\title{
The Effects of Birth Spacing on Nutritional Status in the form of Stunting of Under Five Children in Bangladesh: Evidence Based on BDHS, 2014 Data
}

\author{
Mahfuzur Rahman Khokan* \\ Department of Statistics, Dhaka University, Dhaka-1000, Bangladesh
}

(Received: 9 April 2019 ; Accepted: 21 July 2019)

\begin{abstract}
In this paper, it has been examined the effect of preceding birth interval on nutritional status in the form of Stunting of the children aged under 5 years of age in Bangladesh.For the purpose of the analysis, Bangladesh Demographic and Health Survey (BDHS), 2014 data has been used. The study cases are the children under 5 years aged born during last delivery of their mother. The response variable is Nutritional Status of under 5 year children in the form of stunting. The key independent variables is the length of the preceding birth interval, measured as the number of months between the births of the child under study.Bivariate analysis along with a Chi-square test has been performed to examine the association between preceding birth interval and stunting of the children. To determine the effect of preceding birth interval, three different logistic regression models (Model-I: considering only the preceding birth intervals, Model-II: covariate of model-I along with demographic and socioeconomic factors and Model-III: covariates of model-II along with health related factors) were considered.

The occurrence of stunting has been found to be inversely related with the preceding birth interval for the children aged under 5 years in Bangladesh. This inverse association has been found statistically significant for three models.After adjusting the demographic, socio-economic and health-related factors, the OR (odds ratio) shows that the percentage of being stunted is73\%higher for the children whose preceding birth interval is less than $24(<24)$ while it is $37 \%$ higher for the children whose preceding birth interval is between $24-59$ months compared to whose birth interval is more than $60(>60)$ months with $99 \%$ confidence interval $(1.27,2.36)^{* * *}$ and $(1.07,1.45)^{* * *}$ respectively.
\end{abstract}

Keywords: OR, AOR, Preceding birth Interval, chi-square test, P-value.

\section{Introduction}

Under-nutrition is one of the major problems among children having lifelong consequences not only covering the whole life but transfer from one generation to another ${ }^{1}$. Like other developing counties, it is a common problem in Bangladesh $^{2}$. A child who is malnourished, become a concern to a society in the context of her role as a mother, because being a stunted mother will give birth to a stunted child and the process will rotate.According to World Health Organization (WHO) report ${ }^{3}$, more than half of all child deaths over the world is associated with under-nutrition.

In Bangladesh, millions children are suffering from this nutrition problem namely for stunting from the past to present ${ }^{4}$.It is usually observed that the children are more likely to die from childhood ailments who are malnourished and those who survived, face a recurring sickness and hamper to their growth ${ }^{\mathbf{5}}$. In developing countries, several factors are responsible for the problem under-nutrition among them birth spacing is often overlooked even though having strong relationship with stunting. As a consequence, factors associated with birth spacing are becoming ignored which results some women giving birth interval too short, some for too long and some for just right. It has also been found a clear pattern of increasing chronic malnutrition for having birth spacing shorter, as indicated for all 14 countries by the average of the adjusted odds ratios (OR) ${ }^{6}$.

Some systematic reviews and meta-analysis reveals that short and long spacing between pregnancies has independent impact on increasing risk of adverse maternal, prenatal, infant and child outcomes ${ }^{7}$.A prospective cohort study revealed that the effect of preceding interval were concentrated in early infancy and subsequently in early childhood (ages 1 to 4 years) controlling the effects of child, maternal age, birth order, maternal education, housing area, death of the preceding child, and time ${ }^{8}$. This study also revealed that under-five mortality rate fell down 280 deaths per 1000 births to 174 per 1000 births for the children when the preceding birth interval increases from less than 2 years to 4 or more years. Several studies also has been performed taking the length of the birth interval either dichotomies (short vs. long interval) or a taking log transformation to study young child mortality in relationship to birth interval ${ }^{9-15}$. These studies were multivariate and found significant association between length interval and child mortality.

In this paper, the main concern is to explore whether there is any association between short birth interval with the nutritional outcomes in the form of stunting for children under 5 years considering the effects of demographic, socioeconomic and health related factors on child in Bangladesh.

\section{Data and Methods}

The study used the data of 2014 Bangladesh Demographic and Health Survey (BDHS), a nationally representative sample survey of men and women of reproductive age that provides information on childhood mortality levels, fertility preferences, use of family planning methods, and maternal, child, newborn health and on many other health-related issues $^{16-17}$. In the dataset, the number of cases for the study has been found 3848 who has the information on last born

*Author for correspondence. e-mail: mahfuz_sbi34@yahoo.com 
child for ages under 5 having valid anthropometric information and preceding birth interval. The dependent variable for this study is nutritional status of under 5 children in the form of stunting.

During the DHS survey, the information regarding height and weight of the alive and under five years of aged children were measured along with their respective age to calculate the nutritional status and evaluate against the referenced standard and according to this standard, the nutritional status are considered stunting if the height-forage $\mathrm{Z}$-score is less than $-2 \mathrm{SD}^{18}$.

The main covariate of interest in this paper is preceding birth interval and it is found that shorter birth interval has a strong association with stunting for the children under 5 years of age. The cut-off point for the preceding birth interval has been done as " $\geq 60$ months (ref)", "24-59 months", and "< 24 months". Beside the preceding birth interval, other demographic factors (Sex of child, Age of Child, maternal Age at birth, Region, Residence), socioeconomic factors (Mother's education, Wealth Index, media Exposure) and health related factors (ANC visit, size of child, Duration of breast feeding, maternal height) has also been taken as a consideration to determine whether there is any significant association with the occurrence of stunting.

\begin{tabular}{|c|}
\hline BDIR61FL \\
Cases: 17863 \\
\hline $\begin{array}{c}\text { Last born child for all ages } \\
\text { Cases: } 16079\end{array}$ \\
\hline Cases: 6855 \\
\hline Last born child for ages under five \\
\hline Last born child for ages under five having valid \\
anthropometric information \\
Cases: 6178
\end{tabular}

Fig. 1. Selection of potential cases who have valid anthropometric information with preceding birth interval

To examine the association of preceding birth interval and other factors with stunting, first a bivariate analyzing along with Chi-square test has been done. Since the main exposure variable is preceding birth interval, three multivariate logistic regression model has been performed. These are Model-I: considering only preceding birth interval to find the impact on stunting, Model-II: covariate of Model-I with demographic (Sex of child, Age of Child, Maternal age at birth, Region) and socioeconomic (Mother's education, Wealth Index, Media exposure) factors to examine the effect of preceding birth interval on stunting after controlling the demographic and socioeconomic variables, and Model-III: covariates of Model-II along with health related factors (ANC visit, Duration of breast feeding, Maternal height) to examine the effect of health related factors. To analyze the result, STATA is used.

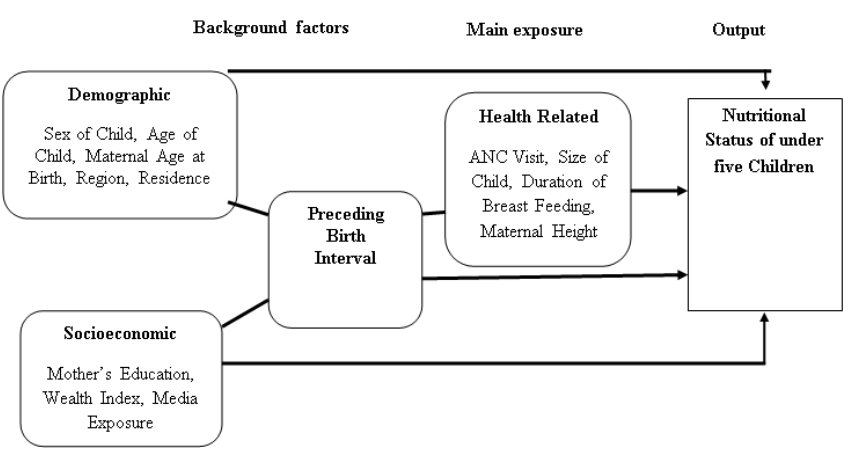

Fig. 2. Conceptual framework based on this study

\section{Results}

\section{Univariate Analysis}

In this section, the factors which are associated with the occurrence of stunting for the newly born child in Bangladesh has been analyzed using BDSH, 2014 data.For this study, we considered 3848 responds who are last born child for ages under five having valid anthropometric information and preceding birth interval.It is observed that the percentage of being an under five children stunted is $38 \%$ and $62 \%$ are not stunted. The preceding birth interval which is the key factor in this paper has three categories showing $42 \%$ of women those were preceding birth interval was more than or equal to 60 months, $47 \%$ was between 24-59 months and only $11 \%$ was less than 24 months. Most of the respondent (76\%) were from rural areas. The gender percentage showed almost equal, 52\% of male and $48 \%$ of female. The maternal age at birth which is also an important factor regarding stunting showed that $97 \%$ of mother were more than 18 year of age. The factor educational status of motherindicates that only $6 \%$ of mother are higher educated, $22 \%$ having no education, $30 \%$ having primary education and highest $42 \%$ having secondary education. It is also observed that $58 \%$ are media exposed and $42 \%$ are not.There are $25 \%$ of the respondents who does not visit for antenatal care, $47 \%$ of them visits 1 to 3 times and $28 \%$ visits more than 4 times. The mother who has height more than $145 \mathrm{~cm}$ are $86 \%$ and $14 \%$ are less than $145 \mathrm{~cm}$.

\section{Bivariate Analysis}

In bivariate analysis, it is observed the association between different determinants withthe occurrence of being a child stunted through the chi-square test. The 
status of different background characteristics by stunting are shown in Table-01.It reveals that $32.8 \%$ children are stunted in case of mother those have the birth interval is more than or equal to 60 months. The percentage of stunting children isshowing increasing pattern when the preceding birth interval decreases. It is observed that $40.04 \%$ children are stunted for the respondents those have received a child after 24-49 months from the previous child and $45.83 \%$ of the children are stunted whose preceding birth interval is less than 24 months. The p-value for the association between preceding birth interval and the children being stunted is 0.0001 which is also a clear indication of statistical significance. It is also observed that the percentage of being stunted is lower $(14.41 \%)$ at the beginning of the age of the child (0-11 months) and it increases with the increase of age and this relation is statistically significant. It is interesting to observe that division has significant relation with the occurrence of stunting for under five children. It shows that the participant who are from Dhaka division has minimum $(29.28 \%)$ percentage of being stunted while Shylet has maximum $(46.07 \%)$. The mother whose maternal age at birth is less than 18 years are more likely $(52.24 \%)$ to give birth a stunted child. Considering mother's education level, higher educated mother's child has less $(20.01 \%)$ chance of being stunted while it is high for the mother who has no education (47.12\%). Among wealth index, poorest group has higher percentage $(47.67 \%)$ of being stunted child and gradually reduced the chance of being stunted with the increase of wealth status showing minimum for the richest group (22.56\%).The percentage of beinga child stunted shows high when there is no antenatal care visit $(45.63 \%)$ and reduces the chance with the increase of the antenatal care visits. It is also interesting to observe that the size of the child at birth has also significant effect on being a child stunted. It says that chance of being a childstunted is high for the child who has the smaller size at birth $(44.16 \%)$ and lesser for those who has average size at birth $(32.99 \%)$.Another important factor is mother's height which has very significant effect of being a child stunted. The mother who are less that 145 $\mathrm{cm}$ of height their children are more prone to be stunted $(54.37 \%)$.

Table 1. Association between selected factors and the percentage of being stunted with p values obtained from Chisquare test statistic.

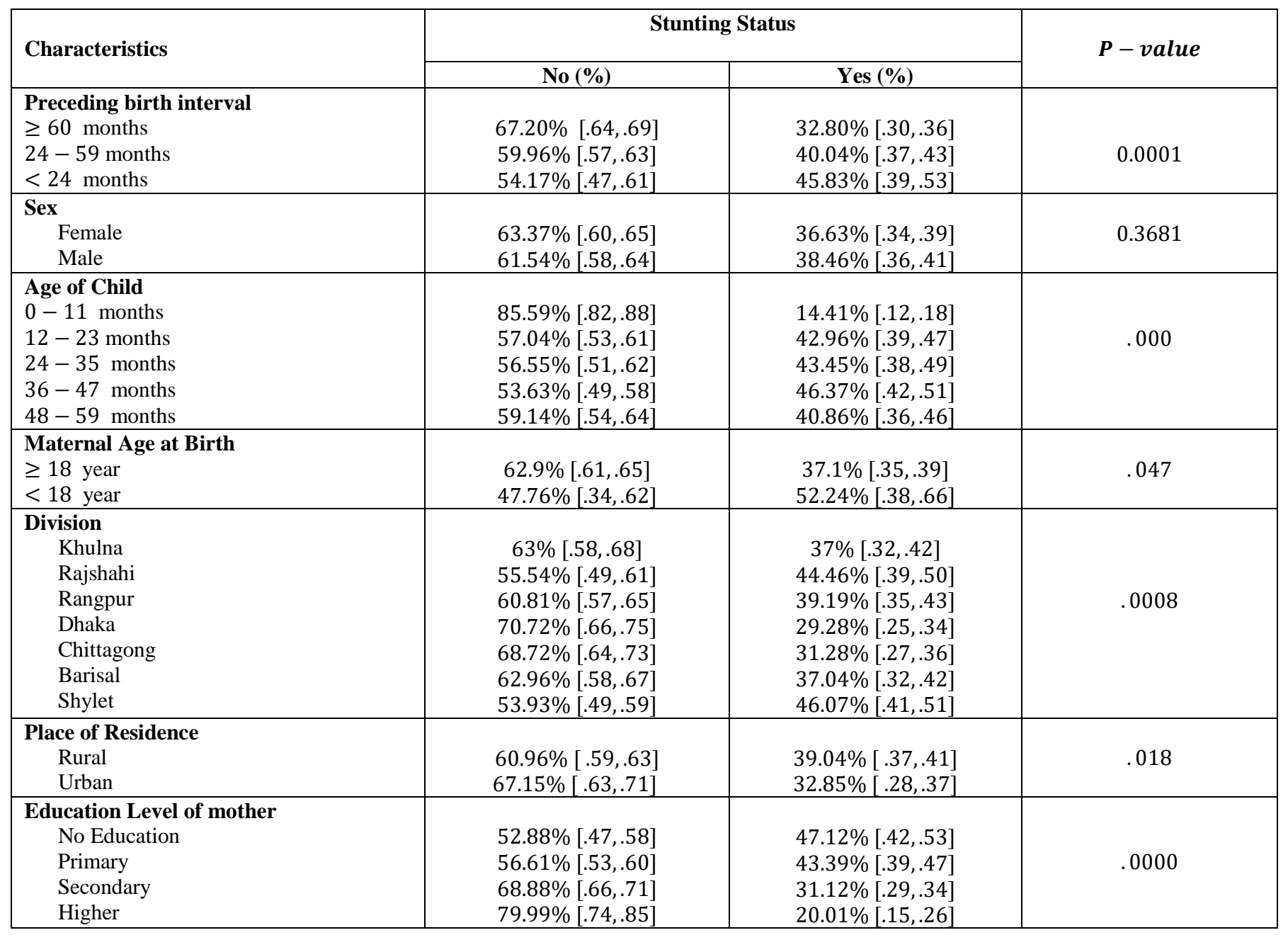




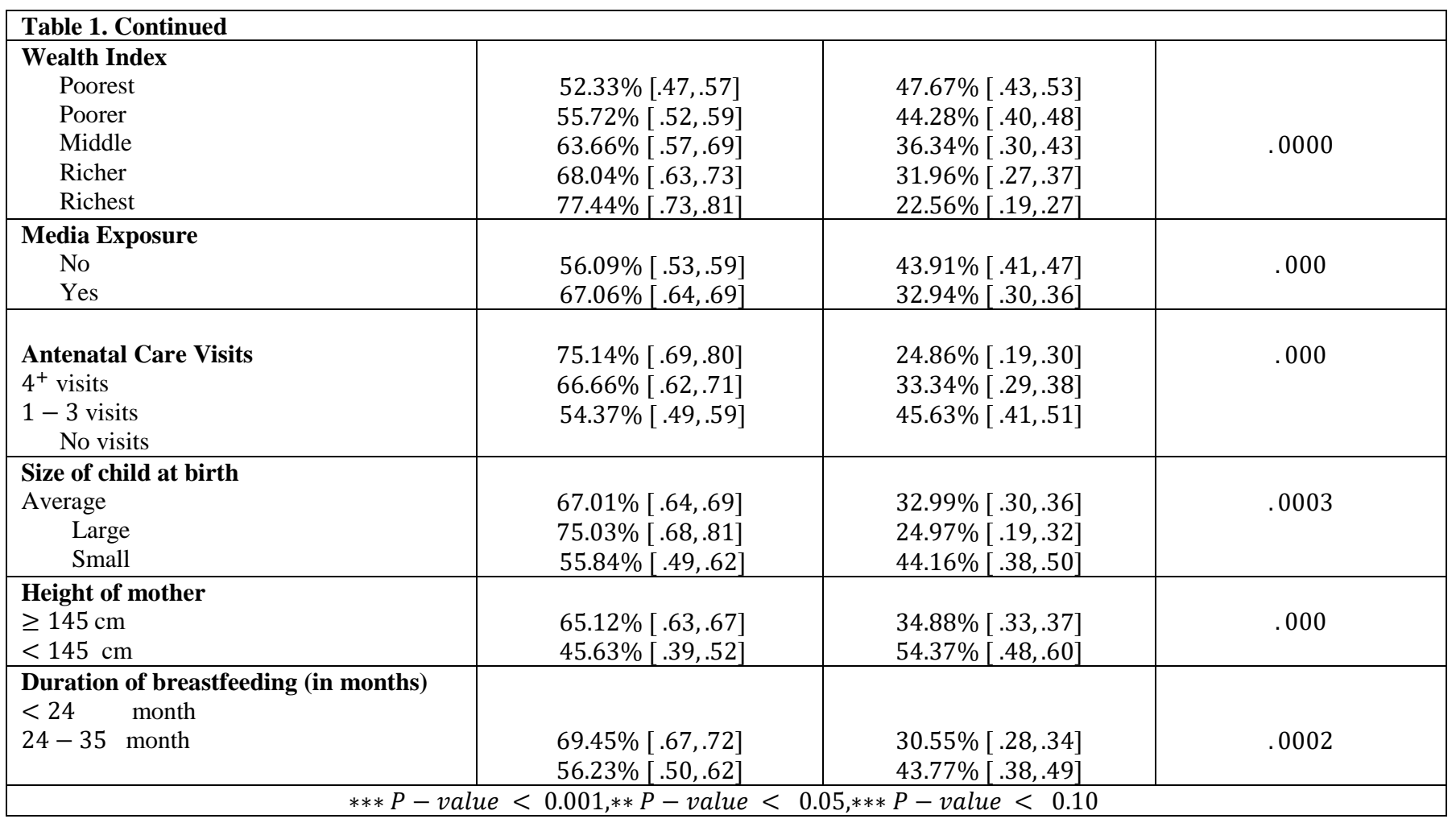

\section{Multivariate Regression Analysis}

Three differentmultivariate logistic regression model has been performed to examine the effect of preceding birth interval on stuntingconsidering other demographic, socioeconomic and health related factors as well and the results are given in the following table-02.It is observed that the preceding birth interval is negatively related with the occurrence of stunting. The first model considered only the effect of preceding birth interval on stunting and it shows that the children whose preceding birth interval is within "24-59" months, are 37\% more likely to happen stunting and if it less than 24 months then it is $73 \%$ more likely to occur stunting. The odds ratios for both categories of precedingbirth intervals ("24-59" months and $<24$ months) are found to be statistically significant at $99 \%$ confidence interval.There are a number of factors that may potentially confounded with the relationship betweenbirth interval and nutritional status of young child.Interestingly it is still observed that the effect of preceding birth interval is negatively relatedwith the occurrence of being a child stunted when the demographic and health related factors has been considered in the model II and model III.The model II considers demographic as well as socioeconomic factors along with preceding birth intervals and it shows that when the preceding birth interval is " $24-59$ " months, then the occurrence of stunting is $22 \%$ higher and $56 \%$ higher for less for 24 months than when it is greater than 60 months at $5 \%$ and $10 \%$ level of significance respectively. In the model III, health related factors has been considered along with the factors considered in the model II and it is also found that the preceding birth interval is inversely related with the occurrence of stunting. The effect of other factors (education level of mother, media exposure, wealth index, maternal age at birth, place of residence, division, antenatal care visits, size of child at birth, duration of breast feeding and height of mother)has also been showed in the table along with corresponding level of significance.

Table 2. Regression coefficients and odds ratios (OR) along with confidence intervals (CI) for the occurrence of stunting obtained from logistic regression model.

\begin{tabular}{|l|c|c|c|}
\hline \multicolumn{1}{|c|}{ Characteristics } & Model-I & Model-II & Model-III \\
\cline { 2 - 4 } & OR (With CI) & OR (With CI) & \\
\hline Preceding birth interval & 1 & 1 & 1.00 \\
$\geq 60$ months & $1.37(1.17,1.59) * * *$ & $1.22(1.03,1.44) * *$ & $1.05(.83,1.35)$ \\
$24-59$ months & $1.73(1.27,2.36) * * *$ & $1.56(1.07,2.27) * *$ & $1.15(.73,1.8) * *$ \\
$<24$ months & & 1 & 1.00 \\
\hline Education Level of Mother & & $0.90(0.68,1.2)$ & $0.87(.66,1.16)$ \\
$\quad$ No Education (Ref) & & $0.64(0.49,0.83) * *$ & $0.72(.53, .99) * *$ \\
Primary & & $0.49(0.32,0.76) * *$ & $.39(.21, .71) * * *$ \\
Secondary & & & \\
Higher & & & \\
\hline
\end{tabular}




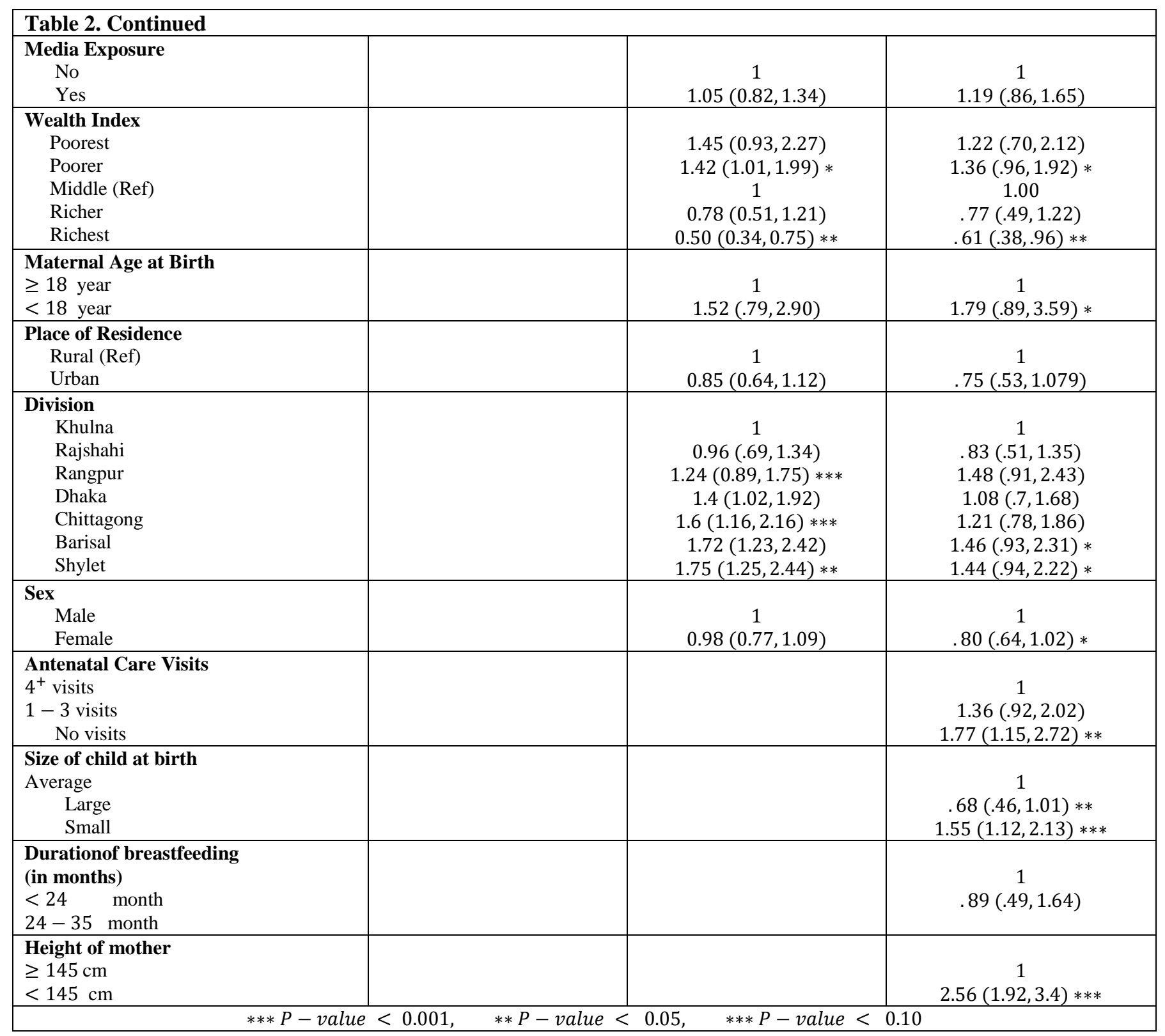

\section{Discussion and Conclusion}

In this paper, the relationship between preceding birth interval and the occurrence of being a child stunted has been analyzed. Firstly, a bivariate analysis has been done to observe the association among the occurrence of stunting along with all associated factors and then a multivariate regression analysis has been performed for stunting considering three logistic regression models. In multivariate regression analysis, firstly it is observed how the unadjusted effects of preceding birth interval associated with the stunting through finding OR (odds ratio) in the logistic regression model-Iand then again observed the effect of preceding birth interval on stunting controlling the demographic, socioeconomic and health-related factors in terms of finding AOR (adjusted odds ratio) in logistic regression model-II and model-III. From both bivariate and multivariate logistic regression model, it is found that the chance of being stunted of children under 5 years of age is negatively associated with preceding birth interval and this association is statistically significant. The three logistic regression model has been performed to show the relationship between preceding birth interval with stunting along with different demographic, socioeconomic and health related factors. Besides the effect of preceding birth interval, it is also observed from the bivariate analysis that educational status of mother, antenatal care visits (4+ visits, 1-3 visits, no visits), region (Khulna, Rajshahi, Rangpur, Dhaka, Chittagong, Barisal, Sylhet), place of residence (rural, urban), wealth index, size of child at birth, height of mother, duration of breastfeeding (in months) are also potential factors associated for the children under 5 years of age being stunted. One of the previous study showed that the chronic malnutrition is significantly linked with shorter 
birth interval.The proportion of being a children stunted was $47.7 \%$ whose preceding birth interval was less than 24 months while it was $45.3 \%$ and $32.5 \%$ for children with a birth interval of 24-48 months and above 48 months respectively.

In this study, it has been found a clear association between preceding birth interval and chance of being stunted for the children under five years of age considering other demographic, socioeconomic and heath related factors. So it is very necessary to take proper steps to enhance the awareness among the people in Bangladesh. It is also important to counselthe married couples of reproductive ages to be more conscious about the necessity of birth spacing and reasons of being a child stunted. As the other factors like region, mother's education, antenatal care visits has clear relation with being a child stunted, so policy makers needs to take different initiatives and should follow up the situation to gain the desired expectation byimproving nutritional status in Bangladesh.

\section{References}

1. Goldenberg RL, JF Culhane, JD Iams, R Romero 2008. Lancet, Epidemiology and causes of preterm birth. 371(9606), 75-84. [PubMed]

2. Liz Young 2002. World Hunger Rutledge Introductions to Development. 20. ISBN 978-1-134-77494-4.

3. World Health Organization. Report of a WHO technical consultation on birth spacing. Geneva, Switzerland, 13-15, 2005 .

4. National Institute of Population Research and Training. Bangladesh demographic and health survey 2007. Dhaka: National Institute of Population Research and Training; 2009, 346.

5. Conde-Agudelo A ${ }^{1}$, Rosas-Bermúdez A, Kafury-Goeta, 2007. Effects of birth spacing on maternal health: a systematic review. Am J Obstet Gynecol. 2007 Apr, 196(4), 297-308.

6. S.O. Rutstein, 2005. Further Evidence of the Effects of Preceding Birth Intervals on Neonatal, Infant, and UnderFive-Years Mortality and Nutritional Status in Developing Countries: Evidence from the Demographic and Health Surveys. Int J Gynaecol Obstet. 2005 Apr;89 Suppl 1:S7-24. Epub 2005 Jan 26.
7. Conde-Agudelo A ${ }^{1}$, Rosas-Bermúdez A, Kafury-Goeta, 2007. Effects of birth spacing on maternal health: a systematic review. Am J Obstet Gynecol, 196(4), 297-308

8. Koenig M, JA Phillips, OM Campbell, S D'Souza 1990. Birth intervals and childhood mortality in rural Bangladesh. Demography, 27(2), 251-65.

9. Miller JE, J Trussell, AR Pebley, B Vaughan 1992. Birth spacing and child mortality in Bangladesh and the Philippines. Demography, 29(2), 305-18.

10. Zenger E. 1993. Siblings' neonatal mortality risks and birth spacing in Bangladesh. Demography, 30(3), 477-88.

11. Alam N. 1995. Birth spacing and infant and early childhood mortality in a high fertility area of Bangladesh: agedependent and interactive effects. J Biosoc Sci., 27(4), 393 404.

12. Alam N, P. David 1998. Infant and child mortality in Bangladesh: age-specific effects of previous child's death. J Biosoc Sci, 30(3), $333-48$

13. Mozumder ABM, KA Barkat-e-Khuda, TT Kane 1998. Determinants of infant and child mortality in rural Bangladesh. ICDDR, B Working Paper, vol. 115. Dhaka (Bangladesh)7 International Centre for Diarrhoeal Disease Research, Bangladesh.

14. Mostafa G, A Foster, V. Fauveau 1995. The influence of sociobiological factors on perinatal mortality in a rural area of Bangladesh. Asia-Pac Popul J 10(1), 63-72 [Bangkok, Thailand].

15. Kathryn G, R Devey, J. Cohen Birth 2004. Spacing Literature: Maternal and Child Nutrition Out Comes. Programme in International Nutrition. University of California, Davis.

16. Hoque ME, MR Khokan, W. Bari, 2014. Impact of stature on non-communicable diseases: evidence based on Bangladesh Demographic and Health Survey, 2011 data, BMC Public Health, 14, 1007

17. Bangladesh Demographic Health Survey Report (BDHS); 2014

18. World Health Organization (WHO). WHO child growth standards: lengths/height-for-age, weight-for-age. Weightfor-length, weight-for-height and body mass index-for-age: methods and development. Geneva: WHO; 2006. 